\title{
CANCHA Y POLIFONÍA: LA NOCIÓN DE ESTILO EN EL CUENTO «YO SOY FONTANARROSA» DE JUAN VILLORO
}

\author{
David GARCÍA CAMES \\ Universidad de Salamanca \\ garciacames@gmail.com
}

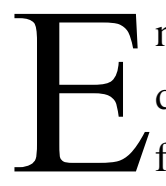

$\mathrm{n}$ el fondo, estamos ante una cuestión de forma, de movimiento en el espacio. Tratar de dilucidar la lógica interna de un gesto que nos define, en el que se revela con mayor o menor fortuna nuestro modo de ser. Dejarse embaucar por el eco de múltiples voces. Ser coherente con la tradición, con quienes nos precedieron, con aquellos que con su sabiduría e intuición marcaron la pauta. Aprender de ellos, imitarlos, innovar a tientas como quien camina en la oscuridad. Respetar su legado pero, precisamente por ello, no poder dejar de aspirar a lo nuevo. Salir al encuentro del otro para descubrir finalmente la voz y el gesto en el que existimos. Narrar y ser narrado. En este artículo hablaremos de fútbol y literatura, de dos manifestaciones que, más allá de los códigos, más allá también de los prejuicios, se basan en la búsqueda del equilibrio, de la precisión, del gesto, de eso que se ha dado en llamar el estilo. Nos referimos a lenguajes, formas de decir, maneras de expresarse que se muestran tanto en el movimiento del futbolista sobre el terreno de juego como en el modo de trabajar la página en blanco del escritor. Ya decía Proust que «la verdad solo empezará en el momento en que el escritor tome dos objetos diferentes, establezca su relación [...] y los encierre en los anillos necesarios de un bello estilo» (1927: 238). Buscaremos, por lo tanto, hasta donde sea posible, las analogías, los nexos entre fútbol y literatura a través de la noción de estilo tomando como punto de partida el cuento «Yo soy Fontanarrosa», de Juan Villoro (Ciudad de México, 1956).

El tratamiento literario del fútbol encuentra en el escritor mexicano a uno de sus principales exponentes. Futbolista frustrado como muchos de los narradores que se han acercado a este deporte desde la ficción, Villoro ha manifestado en diversas ocasiones que «escribir de fútbol es una de las muchas reparaciones que permite la literatura» (2006: 21-22). Ya sea como narrador o cronista, el mexicano encarna a la perfección la posibilidad de emplear el balompié como motivo desde el que trabajar diferentes aspectos del juego que van desde la caída del héroe a la memoria sentimental del aficionado. Autor de relatos futboleros como «El extremo fantasma» (1995) o «El silbido» (2007), así como de los libros de crónicas Los once de la tribu (1995), Dios es redondo (2006) y Balón dividido 
$(2014)^{1}$; el fútbol es para Villoro una pasión anclada en la infancia a la que ha intentado dar una explicación mediante un discurso complejo. Hincha que le va a un equipo sufridor como Necaxa, el escritor ha mostrado asimismo su afición al Barcelona en tanto vínculo con la figura de su padre, el filósofo Luis Villoro, tal y como recordaba en una entrevista: «El primer regalo que recibí de mi padre fue un llavero del F.C. Barcelona [...] Ese llavero me produjo una adicción no solo al fútbol, sino también a frotar las llaves para tratar de concentrarme al escribir» (Cruz, 2012). Fútbol y literatura aparecen ligados a través de este símbolo palpable en la imaginación del narrador mexicano como testimonio de un tiempo que, a pesar de todo, regresa: «Vemos partidos y escribimos de fútbol para recuperar la infancia [...]. El fútbol mejora la infancia que tuvimos del mismo modo en que los sueños permiten que seamos diferentes» (Villoro, 2014: 20).

El cuento que vamos a analizar aquí puede ser leído como el tributo de Villoro a quien fuera uno de sus referentes y uno de los maestros de la denominada literatura del fútbol. La figura del dibujante y escritor rosarino Roberto Fontanarrosa, desdeñada largo tiempo por las elites por su condición de autor de raigambre popular, no ha podido dejar de consagrarse como uno de los grandes nombres de la parodia y el humor en lengua castellana. De él diría el propio Villoro que le parecía casi abusivo que alguien pudiera ser tan bueno como historietista y como escritor (Quiring, 2015). Artesano de la oralidad dotado de un finísimo oído, «El Negro» Fontanarrosa no dejó de mostrarse hasta su último día como un incorregible fanático, llevando el fútbol a sus más altas cotas literarias en algunos de sus cuentos y novelas. El relato «Yo soy Fontanarrosa» fue escrito por Juan Villoro para ser incluido en el volumen colectivo La hinchada te saluda jubilosa ${ }^{2}$, donde autores como el colombiano Daniel Samper o los argentinos Ariel Scher y Jorge Valdano rendían homenaje al rosarino poco después de su muerte, ocurrida el 19 de julio de 2007. El cuento de Villoro también vería la luz en el suplemento cultural del diario La Nación (2007) y en la revista Número O (2009), publicación con vocación periférica dirigida por los escritores mexicanos Guadalupe Nettel y Pablo Raphael. Finalmente el relato sería recuperado por el narrador y cronista en su volumen de cuentos El Apocalipsis (todo incluido) (2014). Antes de pasar a abordar en detalle su trama, tema y motivos, vamos a plantear brevemente algunas ideas en torno a uno de los conceptos sobre los que girará este artículo.

\footnotetext{
${ }^{1}$ A estos libros habría que añadir la correspondencia futbolera que, con el título de Ida y vuelta (2012), Villoro mantuvo con el argentino Martín Caparrós, otro de los autores hispanoamericanos que mejor trabajan el balompié en la actualidad. Entre la obra crítica del mexicano sobre la materia, destacamos el artículo «Los goles y el tiempo», publicado en 1998 en la revista Nueva Sociedad, $\mathrm{n}^{\circ}$ 154, pp. 58-65.

${ }^{2}$ El título del libro está tomado del comienzo del himno de Rosario Central: «Te aplaude y te saluda jubilosa / la hinchada deportiva que te admira. / Campeón de cien jornadas victoriosas, / valiente triunfador que orgullo inspira». Equipo de los amores de Fontanarrosa al que dedicó algunos de sus mejores cuentos, como «19 de diciembre de 1971» 0 «La observación de los pájaros», el escritor siempre participó con devoción de la rivalidad que enfrenta en la ciudad de Rosario a los «leprosos» de Newell's con los «canallas» de Central. Roberto Fontanarrosa terminó por convertirse él mismo en un mito de este equipo, tanto que la hinchada instauró el «Día del Amigo Canalla» para recordar el aniversario de su muerte.
} 


\section{Una cuestión de estilo}

La frase es un lugar común pero, sin embargo, no podemos dejar de hacer mención a ella. Decía Buffon, el ilustrado francés, que el estilo es el hombre. Dice a su vez Buffon, el guardameta italiano, que son las defensas las que hacen buenos a los porteros. El estilo, así en el fútbol como en la literatura, se forja como una dialéctica entre la expresión de la propia voz y su confrontación con la voz del otro. Tanto el futbolista como el escritor precisan encontrar siempre un estilo propio, definido, que les permita ser reconocidos por el lector y el aficionado. El estilo acostumbra a entenderse como expresividad (Ullmann, 1973: 44), forma de manifestación del ser más íntimo que es susceptible de evolucionar con el tiempo. El modo en que un jugador coloque el empeine para adormecer un balón que cae con nieve desde las alturas y depositarlo con mimo sobre el césped será un gesto capaz de revelar su estilo como lo es también la forma en que un narrador escancia entre sus frases el presente de subjuntivo. El estilo es lo que hacemos y aquello en que se muestra lo que somos. Decía Unamuno que «el estilo no se hace. Se nace con él o no se nace. Lo que ocurre es que a las veces tarda uno en encontrar su estilo. O sea, que tarda en encontrarse a sí mismo, en descubrir su propia personalidad» (1924a: 39). En esa búsqueda de un modo de expresión particular el concepto de estilo puede ser compartido por el fútbol y la literatura. Vemos entonces al escritor entregarse al ritmo que le permita encadenar los capítulos de su novela tal como el futbolista precisa de él para mantener la posesión de la pelota en los últimos minutos del partido. El estilo está hecho de cadencia, toque, capacidad de asombro. El estilo, tanto con el balón como con la palabra, es forma, ritmo y seña de identidad. Así lo dejó dicho el editor suizo de origen serbio Vladimir Dimitrijević en su libro La vida es un balón redondo:

El futbolista se descubre de inmediato, no se le puede inventar ni falsificar, es innato; posee un don, un toque inimitable, un arte al amortiguar el balón, algo que no se aprende. Es exactamente como el que tiene un estilo en literatura, pues en mi opinión hay una correlación entre este deporte y la literatura. La manera, para un escritor, de situar una coma, un adjetivo, de entender su propia música, la respiración de su frase, todo eso se encuentra igualmente en este juego mágico. Existe un fútbol musical, jugadores épicos, jugadores líricos, jugadores académicos; se les reconoce tanto en la literatura como en el fútbol (1998: 47-48).

El cuento de Juan Villoro llevará al límite esta analogía. En un ejercicio paródico y humorístico que sin duda le hubiera encantado a Fontanarrosa, el relato arrancará con algunos escritores canónicos jugando al fútbol «en un potrero con más hoyos que pasto» (Villoro, 2007: 59). Por ahí rondan Kafka, Chéjov, Kawabata o Joyce mientras el narrador se arrastra por la cancha con más pena que gloria. Cada uno hará gala de un estilo que armoniza sus virtudes artísticas con las futbolísticas, ofreciendo un relato donde el concepto de equipo participa de una visión fantástica y balompédica de la tradición literaria. Una parodia que se diría delirante donde los recursos de estilo de los escritores se confunden con los gestos técnicos propios del futbolista. A cada literato, de esta forma, se le asignará un lugar en el campo acorde a la imagen arquetípica que de ellos ha quedado fijada en la historia de la literatura ${ }^{3}$. Así, el

\footnotetext{
${ }^{3}$ Ninguno de los escritores que se nombra en este cuento, hasta donde podemos saber, era aficionado al fútbol, salvo Fontanarrosa, claro está. Ahora bien, son muchos los autores que han jugado al fútbol y a los que se les recuerda por ocupar una determinada posición en el campo. El puesto más literario es sin lugar a dudas el de cancerbero, en el que jugaron, entre otros, Albert Camus, Henry de Montherlant, Vladimir Nabokov o Günter Grass. En el Portsmouth AFC jugó de
} 
laconismo y la sobriedad de Chéjov lo llevarán al puesto de mediocentro, «donde hay más gente para dar consejos», del mismo modo que la sutileza de Kawabata se traducirá en la fina estampa de un lateral izquierdo que «disparaba diagonales imprevistas» (2007: 60). Moviéndose entre la transducción y el cliché, nos encontraremos asimismo con Joyce, ese «presuntuoso que se sentía hecho a mano» (2007: 60), esperando en la punta de ataque los pases de Cortázar, fantasista y número 10 del equipo, encarnación de aquellos jugadores para los que, como sostiene Villoro en uno de sus ensayos, «meter un gol es una vulgaridad que hay que dejar en pie de los artilleros» (1995: 58).

$\mathrm{El}$ arranque del cuento «Yo soy Fontanarrosa» se nos ofrece así como obertura en la que resuenan las voces de estos escritores llevados a la cancha, polifonía de estilos que se distribuyen alrededor de la pelota. Dispuestos en un clásico dibujo táctico de 4-4-2 con rombo en el centro del campo -si bien existen un par de posiciones que no se especifican en el relato $-{ }^{4}$, la fuerza y sobriedad de Hemingway se traducirá, por ejemplo, en su puesto de guardameta, del mismo modo que el «porte» de Tolstoi y la «facha de basquetbolista» (2007: 60) del nigeriano Ben Okri los situarán totémicos en el centro de la zaga. Bajo el mando y «el pésimo humor» de Kafka, capitán del equipo, todos ellos tratarán de detener sin éxito las continuas embestidas del contrario. Dice Steiner que «todo estilo literario es un lenguaje dentro del lenguaje» (1967: 306). En cierta forma, es precisamente eso lo que nos encontramos aquí, una combinación de usos estilísticos, una estructura fugada de lenguajes y modos de expresión inalienables que se combinan a través de la fusión de fútbol y literatura. Convertidos en futbolistas y material literario, Villoro se sirve de estos narradores para dar rienda suelta a un juego con la tradición al más puro estilo de Fontanarrosa. Revisitar el canon, subvertirlo, entregarse a la parodia. Se diría que, en cierta medida, estamos ante el sueño de la Weltiteratur de Goethe reducido a una selección mundial de escritores que deambulan por el campo ${ }^{5}$. Sin embargo, la voz del narrador será la encargada de despertarnos muy pronto de esta quimera.

\section{Al primer toque}

Uno de los recursos de estilo más empleados por Fontanarrosa en sus cuentos era, sin lugar a dudas, la vuelta de tuerca, el repentino cambio de sentido de un relato manifestado en un giro

\footnotetext{
portero y lateral derecho Arthur Conan Doyle. El argentino Osvaldo Soriano era un prometedor delantero en los juveniles de San Lorenzo de Almagro hasta que una lesión de rodilla truncó su carrera. El propio Villoro estuvo en las divisiones inferiores del conjunto mexicano de los Pumas. Pero, sin duda, el caso más curioso y disonante es el de Miguel Hernández, extremo derecha en un equipo aficionado de Orihuela llamado La Repartidora.

${ }^{4}$ El texto de Villoro distribuye los jugadores de la siguiente forma: Hemingway se sitúa de portero tras una defensa de cuatro formada por Fontanarrosa como lateral derecho, Tolstoi y Ben Okri de centrales, y Kawabata de lateral izquierdo; en el centro Chéjov es el 5 y organizador, Kafka está a su lado si bien no sabemos la posición exacta, con Cortázar en lo alto del rombo como mediapunta; Joyce queda arriba como delantero centro, aunque desconocemos quién es su compañero de ataque. A pesar de que, como las revoluciones científicas y los paradigmas literarios, las tácticas futbolísticas cambian con el paso de los años, el 4-4-2 permanece como uno de los dibujos más utilizados. Para saber más puede consultarse el libro de Jonathan Wilson (2008): La pirámide invertida. Historia de la táctica en el fútbol. Madrid, Debate, 2013.

${ }^{5}$ Los británicos Monty Python ya crearon en su programa Monty Python's Flying Circus (1969-1974) un sketch bajo un esquema similar al de Villoro. En este caso se trataba de un partido de fútbol filosófico entre Grecia y Alemania arbitrado por Confucio, San Agustín y Santo Tomás de Aquino. El sketch «International Philosophy» (1972) puede verse aquí: https://www.youtube.com/watch?v=hReASuvB92U (última consulta, 21-7-2017).
} 
sorprendente de la trama. Juan Villoro también parece rendir homenaje al argentino en este punto cuando, tras habernos sumergido en un arranque presidido por los célebres escritores en la cancha, nos explica lo que se oculta tras ese alucinógeno partido de fútbol. El narrador nos entrega en primer término un retrato de sí mismo donde se define como un escritor que en el potrero despliega «un fútbol de clase que no siempre se aprecia» (Villoro, 2007: 60), si bien durante el partido no hace más que suceder sus errores uno tras otro fruto de su falta de práctica y su pésimo estado físico. La voz principal del relato revelará al poco tiempo que se halla jugando por su libertad en un encuentro completamente real: «Nuestro equipo llevaba nombres de escritores en los dorsales. Eso era especial. Más especial era que mis diez compañeros trabajaban en la policía» (2007: 62). Es decir, el punto de vista de la primera persona nos permite abandonar de forma inesperada el sentimiento de lo fantástico para embarcarnos en una narración casi costumbrista. Lo que se antojaba un puro ejercicio lúdico con el canon toma el aspecto de una broma donde, sin saberlo, el lector también estaba incluido. Los escritores mantienen su nombre pero devienen policías mientras nos vemos obligados a aceptar el cambio radical en la atmósfera del relato.

El protagonista empieza a dar a conocer entonces algunos detalles sobre la tragicómica historia que le ha llevado a esta situación. Sabemos que se trata de un escritor con ínfulas de incomprendido su estilo en el campo de fútbol también lo delata - que se dirige a Ciudad Moctezuma acompañando a un amigo que busca un «mecánico baratísimo» para cambiar el claxon de su coche. El viaje por los andurriales del estado de Sonora, con esos dos tipos a la deriva, no puede evitar remitirnos a los escenarios y motivos de Bolaño. Apremiado y desesperado, el narrador se parará a orinar en un lugar de la carretera, miccionando sin querer sobre una estatua de Benito Juárez, alevoso atentado contra el prócer que provocará su arresto: «Orinar una propiedad (ajena) es delito. Mancillar un símbolo patrio es un delito peor» (Villoro, 2007: 64). Los policías, «juaristas convencidos», lo conducirán finalmente a un campo de fútbol tras recibir un aviso por radio. Descubrimos entonces los detalles y el origen de esa extraña pachanga literaria:

-Nos falta un jugador -me explicó el policía que me había arrestado.

Fue así como me entregaron la camiseta de Fontanarrosa.

-Para ponértela, tienes que aprender esto -me dieron una tarjeta.

El ayuntamiento izquierdista había lanzado un peculiar programa de promoción de la lectura entre los policías. Les daba uniformes a condición de que portaran nombres de escritores. Para vestir la camiseta, había que saber quién era el autor que la respaldaba. Después del partido se celebraba una velada literaria (2007: 65-66).

Una vez cerrada la analepsis, volvemos a situarnos en el instante inicial del cuento, con el narrador recibiendo una reprimenda de Kafka en el terreno de juego. Jugando de lateral derecho y portando la camiseta de Fontanarrosa, un escritor que como veremos no es muy de su agrado, el protagonista terminará por ser expulsado del partido después de una feísima entrada sobre el extremo izquierdo del rival. El hecho de abandonar el registro fantástico no hace sino ahondar en el cariz humorístico del relato. La tensión a la que se ve sometido el protagonista en su arresto, junto con su falta de maña para jugar al fútbol, propiciarán toda una serie de situaciones grotescas donde no faltan 
los goles en propia puerta, las amenazas de Hemingway o el sadismo del árbitro (2007: 67). Sostiene el crítico argentino Pablo de Santis al hilo de la obra cuentística de Fontanarrosa que la parodia no solo «le permite la aparición de lo específicamente humorístico, sino también le presta un orden a la narración» (2000: 501). El cuento de Villoro responde sin lugar a dudas a esta premisa, empleando elementos paródicos y recurriendo al humor como elemento cardinal del relato. El tributo del escritor mexicano se manifiesta, por lo tanto, a través de algunos de los principales rasgos de estilo de Fontanarrosa, como pueden ser los giros coloquiales de la lengua, el sorprendente sentido de la oralidad, el uso continuo de la ironía o la manera de sortear las fronteras entre las consideradas alta y baja cultura.

Esto también resultará evidente en el encuentro final del narrador con Jorge Linares, un escritor admirado por los policías que acudirá a la tertulia literaria posterior al partido. Viejos compañeros de letras, el narrador y Linares entablarán una conversación donde, a través de sus opiniones sobre Fontanarrosa, podemos entrever la estética a la que se adhiere el autor. Mientras el protagonista confiesa que hace años escribió una crítica negativa sobre el argentino porque «quería mostrarme como escritor sofisticado y no me pareció correcto elogiar a un caricaturista» (2007: 66); Linares, a quien en algunos aspectos podemos considerar un trasunto del propio Villoro, manifestará su admiración incondicional hacia «El Negro». La ridícula pedantería de la voz principal se expresará en el resentimiento que siente hacia Linares, escritor que, en sus propias palabras, «adquirió la deleznable notoriedad de un cronista de fútbol» (2007: 69). Volviendo de nuevo a la cancha, podemos plantear que la disputa entre estos dos escritores resulta equiparable a la dialéctica entre dos formas de entender el fútbol. Por un lado, se diría que el narrador encarna la literatura «resultadista», aquella que busca la crítica complaciente y que en su ensimismamiento deja de lado la diversión; mientras que la voz de Linares parece abogar por la literatura de «juego bonito», aquella capaz de encandilar al lector al primer toque y llevarlo a morir de risa en el sofá, tal y como pretendía Fontanarrosa con sus cuentos. El relato se cerrará finalmente con un juego de espejos en que el narrador confiesa haber cedido a Linares su historia a cambio de su libertad: «El hijo de puta se quedó con mi cuento. No digo que yo lo hubiera escrito como Borges, pero sí como un mejor escritor del Occidente. Modestia aparte, él tiene el tema, pero no tiene mi voz» (2007: 70).

\section{Comparatismo futbolero}

Fútbol y literatura aparecen en este relato como dos disciplinas que, lejos de mostrarse antagónicas, se entrelazan en la narración dibujando un peculiar contrapunto. Sin abandonar en ningún momento la ironía, pasamos de una a otra con la soltura con que mueven la pelota en el centro del campo determinados equipos, casi sin darnos cuenta, dejándonos llevar por la fluidez y, si se quiere, verticalidad del discurso del narrador. Fútbol y literatura, lejos de contraponerse según sostienen los tópicos, se citan aquí mostrando las posibilidades latentes en el tratamiento literario del balompié. En el fondo, pensándolo bien, nos hallamos frente a un ejercicio de estilo basado en una suerte de 
comparatismo futbolero. Si adoptamos la definición clásica de Remak de literatura comparada como «la comparación de una literatura con otra u otras y la comparación de la literatura con otros ámbitos de la expresión humana» (1961: 89), veremos que, más allá de los muchos matices terminológicos que puedan plantearse, este concepto ampara la capacidad de establecer un nexo entre la literatura y el fútbol como objeto de análisis. Relacionar diferentes esferas de la creatividad humana como forma de iluminar y descubrir nuevos significados en cada una de ellas. Es preciso, por tanto, salir al encuentro de aquellas relaciones estructurales (Wellek y Warren, 1948: 155) que no tendrían por qué limitarse a las expresiones humanas consideradas puramente artísticas; es preciso, como hace Juan Villoro en este relato, buscar los puntos en común, jugar con ellos, así en la ficción como en la crítica.

El fútbol, fenómeno de masas global como ningún otro a lo largo de la historia, puede llegar a ser entendido como una lengua franca compartida por tres cuartas partes de los habitantes del planeta. Como le sucede al narrador del cuento de Villoro, resulta difícil no sucumbir a esa fascinación infantil de ver niños jugando en un parque y patear con ellos la pelota aunque uno le pegue «al carrito de los algodones de azúcar» (2007: 62). El fútbol establece una comunicación inmediata alrededor del influjo mágico de una esfera, lenguaje vehicular cuyos gestos se traducen al instante. Deporte y relato que continuamente demanda el empleo de la palabra, el fútbol se manifiesta en la literatura a través de un universo simbólico donde resuenan toda una serie de mitos fijados en el imaginario colectivo ${ }^{6}$. La literatura de ficción encuentra su caladero dentro del mundo del fútbol en ese lugar donde los episodios de la cancha aciertan a traducir metáforas esenciales sobre el ser humano. Es precisamente ahí donde el comparatismo puede moverse con mayor desenvoltura, ofreciendo nuevos sentidos en la convergencia de ambas disciplinas. Si entendemos la literatura como un conjunto de voces, no podemos dejar de leer este texto de Villoro como una polifonía donde, además de darse cita algunos de sus referentes, también se encuentra plasmado su ideario estético sobre el fútbol. De la tensión entre fútbol y literatura, de su resolución en el relato, surge una forma original que parece encajar con lo que propugnaba el escritor argentino Juan Sasturain en un artículo:

Tanto la práctica del fútbol [...] como el ejercicio de la literatura [...], llevados a su grado de excelencia y respeto por los medios y posibilidades, pueden (aunque no suelen) alcanzar el grado de la artisticidad: pueden ser un arte, no sólo una actividad reglada por la eficacia o un trabajo marcado por la recompensa. El manejo de la pelota como el del lenguaje - puestos en buenos pies y manos- son un desafío a la creatividad y de ahí, de esa tensión por encontrar una forma original, cada vez única, para resolver dificultades expresivas, puede saltar la belleza. Ambas actividades tienen en común su condición de juego en tanto desafío, actividad en el fondo inmotivada, asunción de un riesgo y entrega personal (2012).

Esta búsqueda de la armonía que, en sus representaciones ideales, comparten fútbol y literatura, propicia un vínculo hasta cierto punto interartístico donde ambas manifestaciones comparten algunos de sus códigos: «El ansia de "jugar bonito" también tiene su origen en el nivel trascendental. Es un ansia que nace de una sublimación estética» (Ilundáin-Agurruza, 2006: 48). Pensamos en la rápida circulación del esférico, en un juego vistoso consagrado al espectáculo, así como leemos las frases

\footnotetext{
${ }^{6}$ Esta es la idea principal de mi tesis de doctorado La jugada de todos los tiempos: mito y fútbol en la literatura hispánica, presentada en febrero de 2017 en la Universidad de Salamanca y dirigida por los profesores José Antonio Pérez Bowie y Javier Sánchez Zapatero.
} 
cortas y el ritmo ágil del relato de Villoro: «Ya sin mí, el equipo recibió otros dos goles, pero ellos no reconocieron que les hice falta. Después de los tres pitidos finales, volvieron a verme con ojos de sacrificio mesoamericano» (2007: 67). El narrador y cronista de Ciudad de México ha buscado conciliar en este texto dos de sus pasiones, ha querido divertirse escribiendo un cuento que en el fondo es puro juego. Nada queda al margen de la parodia. Ahí tenemos, por ejemplo, una burla al dogmatismo de los estudios post-coloniales cuando el narrador afirma que su equipo cuenta con Ben Okri porque está de moda tener africanos o bien recuerda que acusó en una reseña a Fontanarrosa de ser «colonialista por escribir “mejicano" en vez de "mexicano"» (2007: 69).

\section{La necesidad de la polivalencia}

El relato de Juan Villorose encuentra permeado de forma notable por las nociones de influencia e intertextualidad. Resulta imposible dejar de leer este texto como un «mosaico de citas» (Kristeva, 1969: 190) procedentes tanto de la cancha como de los anaqueles. Entre todas ellas destacará, como ya hemos apuntado, la referencia continua a Fontanarrosa, origen y lector in fabula del cuento. Desde el mismo título al constante humor, el escritor rosarino sobrevuela cada una de estas páginas. El homenaje se hace patente sin ir más lejos en esa consideración del balompié como pasión irredenta a la que resulta casi imposible conceder una explicación racional. Dice en un momento el narrador, respondiendo a un policía y parafraseando a Amiel, que «el fútbol es un estado de ánimo» (Villoro, 2007: 65). Ese era ciertamente el sentir del propio Fontanarrosa, capaz de explicar como nadie las soledades y éxtasis del hincha y capaz de vivirlas en su propia piel sin atisbo de impostura. Recordemos, por ejemplo, el final de uno de sus cuentos más célebres, «Memorias de un wing derecho», donde se ve obligado a recurrir a la tautología para explicar la intensidad de esa locura nacida de un potrero: «Porque el fútbol es el fútbol. Esa es la única verdad. ¡Qué me vienen con esas cosas! Son modas que se ponen de moda. Y después pasan. El fútbol es el fútbol, viejo. El fútbol. La única verdad. ¡Por favor!» (Fontanarrosa, 1983: 164).

El diálogo entre los textos del discípulo y el maestro se expresa a su vez en la capacidad de ambos para moverse con total naturalidad entre las esferas de la llamada alta cultura y la cultura de masas. El mismo hecho de que uno de los principales autores hispanoamericanos de la actualidad escriba un cuento a partir del supuesto de que varios escritores canónicos jueguen un partido de fútbol sería imposible sin la obra precedente de escritores como el propio Fontanarrosa, Osvaldo Soriano, Mario Benedetti, Manuel Vázquez Montalbán o Augusto Roa Bastos, capaces de franquear tabús y componer algunas de las mejores piezas de la literatura balompédica. El fútbol aparece legitimado como material literario desde el momento en que el autor tiene suficiente talento para trabajarlo y llevarlo mucho más allá de esa «cháchara deportiva» ${ }^{7}$ con la que nos abruman los medios de

\footnotetext{
${ }^{7}$ Este término procede de un artículo de Umberto Eco con el mismo nombre: «La cháchara deportiva, nacida como elevación a la enésima potencia de ese derroche inicial (y razonado) que era el juego deportivo, es la magnificación del Derroche, y por tanto el punto máximo del Consumo. Sobre ella y en ella, el hombre de la sociedad de consumo se consume a sí mismo» (1969: 186-187).
} 
comunicación día tras día. No es el tema el que determina la categoría de una obra sino la forma en que el artista lo fija en su texto. Como el futbolista de largo recorrido capaz de desempeñarse en diferentes posiciones del campo, el escritor también precisa polivalencia para saber encontrar un término medio entre lo culto y lo popular: «Siempre he respetado los códigos del fútbol, pero no me gustaba que un tipo con pelo de roedor (de hámster, para ser exacto) pusiera en entredicho su autoridad haciéndole caso a Chéjov, que me ordenaba como si fuera Johan Cruyff» (2007: 61). El escritor mexicano muestra en fragmentos como este su capacidad para entretejer una trama con los mimbres del fútbol y la literatura, para transitar de uno a otro lado, para adherirse, de alguna manera, a esa reconciliación de la que hablaba Claudio Guillén cuando se refería a «la conjunción de lo uno y lo múltiple; el azar, el accidente, el acontecimiento singular; sin doblegarlos a una lógica disyuntiva, reduccionista y unidimensional» (1995: 66).

\section{El legado y el estilo}

Llegados a este punto, tenemos a un burlador de épicas y solemnidades llamado Roberto Fontanarrosa homenajeado por uno de sus continuadores, el mexicano Juan Villoro. El fútbol es el lugar común, casi podríamos considerarlo el estilema ${ }^{8}$, que los reúne en este relato donde se concitan el humor y la parodia. Un delirante equipo conformado por escritores nos ha permitido plantear la posibilidad de trabajar la noción de estilo en dos disciplinas tan aparentemente dispares como el fútbol y la literatura. Quizá debamos hacernos perdonar el hecho de haber incurrido en el pecado de la caza de analogías, pero creemos, por el contrario, que es preciso abrir nuevas vías de análisis, explorar terrenos casi yermos en los que nuestra sociedad también se revela. El estilo ha sido aquí la clave en que armonizar las diferentes voces de la polifonía que se oculta tras todo texto. Ello nos ha permitido comprender que en la cancha el puesto idóneo para Tolstoi es el de defensa central, del mismo modo que Chéjov puede revelarse como un magnífico mediocentro. Signos lingüísticos y habilidades futbolísticas terminan por confundirse plenamente en esta broma literaria de Villoro. El estilo, entendido como expresividad, como manifestación de la personalidad a través de diferentes medios, se muestra así como punto de unión, vínculo entre aquello que somos y decimos: «Estilo es lo que da unidad o, mejor aún, la "unicidad" de una obra literaria» (Alonso, 1998: 115).

Para encontrar la voz, para descubrir nuestro estilo, resulta imposible dejar de escuchar las voces que nos rodean. Estamos hechos del eco de la otredad que sin cesar resuena y nos moldea. Villoro necesitó a Fontanarrosa como Messi a Maradona y el crítico a su maestro. El otro nos revela, sin ir más lejos, esa fascinante capacidad de prestar oído a todo lo que sucede alrededor, esa amplitud de miras que le permite estar al tanto de la más pequeña novedad en su campo de estudio, provenga de donde provenga. El maestro es aquel capaz de jugar con la alta cultura y la cultura de masas merced a su extrema honestidad y su abrumador sentido de la ironía. En el placer de haber compartido y seguir

\footnotetext{
${ }^{8}$ Tomamos la definición de estilema de Lázaro Carreter en su Diccionario de términos filológicos: «Término alguna vez empleado para designar un rasgo o una constante de un estilo» (1968: 172).
} 
compartiendo su voz aprendemos a forjarnos a nosotros mismos: «El estilo se va haciendo, y es porque el artista se está buscando a sí mismo [...]. Y cuando se encuentra es que ha encontrado su obra; es que su obra le ha hecho a él» (Unamuno, 1924b: 101). La obra nos dice, nos habla, y en su legado aprendemos a reconocernos. Admiramos la clase y el estilo que permiten a Villoro y Fontanarrosa jugar con el fútbol y la literatura, tal como admiramos la sabiduría de quien es capaz de transitar de unos versos en latín de Virgilio al último capítulo de Bob Esponja con la más absoluta naturalidad. El estilo es forma, modelo e intuición de aquello que se nos escapa: «Nuestra vida es también la vida de los demás; pues, para el escritor, el estilo es como el color para el pintor, una cuestión no de técnica, sino de visión» (Proust, 1927: 245). Es necesario, pues, mirar al otro lado, descubrir en el homenaje que hemos analizado otro homenaje más sincero si cabe. El juego es lícito, consecuente. Y si alguien se decide a cambiar, aunque sea por un segundo, la lectura de Miajil Bajtin por la biografía de Andrés Iniesta nos daremos por satisfechos.

\section{Referencias bibliográficas}

Alonso, Dámaso (1998): Motivación y arbitrariedad del signo lingüístico. Málaga, Analecta Malacitana.

CRUZ, Juan (2012): «Mi pasión empieza con un llavero del Barça. Entrevista a Juan Villoro», El País, 4 de marzo, en http://deportes.elpais.com/deportes/2012/03/04/actualidad/1330887744_389311. html (última consulta, 25-6-2017).

DE SANTIS, Pablo (2000): «Risas argentinas: la narración del humor», en Noé JiTRIK, ed., Historia crítica de la literatura argentina XI. Buenos Aires, Emecé, pp. 493-510.

DimitriJeviĆ, Vladimir (1998): La vida es un balón redondo. Trad. de Antonio Castilla Cerezo. Madrid, Sexto Piso, 2010.

ECO, Umberto (1969): «La cháchara deportiva», en La estrategia de la ilusión. Trad. de Edgardo Oviedo. Barcelona, Lumen, 1996, pp. 182-187.

FONTANARROSA, Roberto (1983): «Memorias de un wing derecho», en Cuentos reunidos 1. Madrid, Alfaguara, 2003, pp. 161-164.

GuILlÉN, Claudio (1995): «Lo uno con lo diverso: literatura y complejidad», 1616: Anuario de la Sociedad Española de Literatura General y Comparada, vol. IX, pp. 51-66.

ILUNDÁIN-AgurRuZA, Jesús (2006): «Goles trascendentales», en César R. TORRES y Daniel G. CAMPOS, eds., ¿La pelota no dobla? Ensayos filosóficos en torno al fútbol. Buenos Aires, Libros del Zorzal, pp. 25-58.

KristeVA, Julia (1969): Semiótica 1. Trad. de José Martín Arancibia. Madrid, Fundamentos, 1978. LÁZARO CARRETER, Fernando (1968): Diccionario de términos filológicos. Madrid, Gredos, 1990.

Proust, Marcel (1927): El tiempo recobrado. En busca del tiempo perdido VII. Trad. de Consuelo Berges. Madrid, Alianza, 2008. 
230 Tropelías. Revista de Teoría de la Literatura y Literatura Comparada, número extraordinario 2 (2017) David García Cames

QUIRING, Débora (2015): «Su mundo y el nuestro. Juan Villoro recibirá el premio José Emilio Pacheco», La Diaria, 11 de noviembre, en https://adiaria.com.uy/articulo/2015/11/su-mundo-yel-nuestro/ (última consulta, 23-6-2017).

REMAK, Henry H. H. (1961): «La literatura comparada: definición y función», en María José VeGA y Neus CARbOnell, eds., La literatura comparada: principios y métodos. Trad. de María José Vega. Madrid, Gredos, 1998, pp. 89-99.

SASTURAIN, Juan (2012): «Con las palabras a la cancha», Página/12, 10 de abril, en http://www.pagina12.com.ar/diario/contratapa/13-191501-2012-04-10.html (última consulta, 22-6-2017).

STEINER, George (1967): Lenguaje y silencio. Trad. de Miguel Ultorio, Tomás Fernández y Beatriz Eguibar. Madrid, Gedisa, 2006.

Ullmann, Stephen (1973): Significado y estilo. Trad. de Juan García-Puente. Madrid, Aguilar, 1979. UnAmuno, Miguel de (1924a): «Estilo y pluma», Los Lunes de El Imparcial, 27 de abril, en Alrededor del estilo. Ed. de Laureano Robles. Salamanca, Ediciones Universidad de Salamanca, 1998, pp. 37-39.

(1924b): «El estilo nos hace», Los Lunes de El Imparcial, 24 de agosto, en Alrededor del estilo.

Ed. de Laureano Robles. Salamanca, Ediciones Universidad de Salamanca, 1998, pp. 101-103.

VILLORO, Juan (1995): Los once de la tribu. México, Aguilar.

- (2006): Dios es redondo. Barcelona, Anagrama.

(2007): «Yo soy Fontanarrosa», en Luciana MAINELLI, ed., La hinchada te saluda jubilosa. Rosario, Fundación Ross, pp. 59-70.

- (2014): Balón dividido. México, Planeta.

Wellek, René - Warren, Austin (1948): Teoría literaria. Trad. de José María Gimeno. Madrid, Gredos, 1985. 